Ljubiša Garić

https://doi.org/10.21278/TOF.41303

ISSN 1333-1124

eISSN 1849-1391

\title{
ANALYSIS OF VIBRO-IMPACT PROCESSES OF A SINGLE-MASS SYSTEM WITH VISCOUS DAMPING AND A SINGLE LIMITER
}

\begin{abstract}
Summary
The paper presents an analysis of the horizontal straight-line motion of a single-mass, one-sided vibro-impact system in the cases when the external coercive force and the viscous damping force are known and a periodic vibro-impact mode is realized in the system. Vibroimpact systems represent the basis of industrial machinery. Periodic motion modes, realized as forced damped oscillations, are characteristic of these systems. Due to the variety of vibroimpact systems, there are various computing methods used for the analysis and description of processes in vibro-impact systems. The process of obtaining results is, generally, very complex and time-consuming because there is a need of using linear differential equations in computing. The aim of the analysis presented in this paper is to determine the areas of the periodic vibro-impact mode existence.
\end{abstract}

Keywords: $\quad$ vibro-impact oscillator, vibro-impact modes, external coercive force, viscous damping force.

\section{Introduction}

The subject of the research conducted in this paper is the analysis of the horizontal straight-line motion of a single-mass, one-sided vibro-impact system, when a periodic vibroimpact mode is realized in the system. This analysis is conducted using mathematical modelling of vibro-impact sytems. For this purpose, it is assumed that the external coercive force and the viscous damping force are known, while it is necessary to determine conditions (areas) of the periodic vibro-impact mode existence. Two examples (cases) are explored: the first, when the restitution coefficient is $\mathrm{R}=0.7$, damping exists, and the damping decrement is $\Delta_{x} \neq 0$; the second, absolutely elastic impact, with the restitution coefficient $\mathrm{R}=1$ and with no damping, $\Delta_{x}=0$. In both cases, results enabling the stability of vibro-impact system motion are obtained. The aim of the performed research is an increase (improvement) in the periodic vibro-impact system functional efficiency, based on the mathematical model of the system.

This paper considers and presents the results obtained for a defined form of the vibroimpact system structure (with a single-mass, a single limiter and one spring), which is exposed to the influence of a periodic external force. The obtained results can give some ideas and guideposts for research into vibro-impact systems with different structures and different types of external force. 
The most important scientific results which improve the knowledge about the dynamics of vibro-impact systems have been published in scientific literature, most notably in [1], [2] and [3]. In these publications, the analysis of vibro-impact systems is performed assuming that system parameters and the external coercive force are known; subsequently, corresponding modes are defined and conditions for the existence of these modes are examined. In [1], Babickii V.I. examined the existence of the periodic vibro-impact system mode for the time interval with a defined period of oscillation. This was a guidepost which was followed in the research presented in this paper. Characteristics of the horizontal straightline motion of single-mass, one-sided vibro-impact systems are presented in [4], [5], [6], and [7]. A vibro-impact system with two masses moving separately and impacting mutually is presented in [8], while a vibro-impact system with a movable limiter is presented in [9]. Stability considerations of the periodic motions of vibro-impact systems are given in [10]. In [11], vibro-impact systems with a single limiter as well as with two limiters fixed symmetrically on both sides of the oscillator are presented and described. The example presented in [12] is a systematic study on a periodically forced oscillation system with impact.

Vibro-impact systems are widely used in mechanical engineering. Usually, periodic vibro-impact systems are used when time intervals between two consecutive impacts are the same. Under this condition, impacts are performed during the forced system motion, which produces various effects. Dynamic phenomena occurring as the result of impact can produce harmful or beneficial effects. The harmful effects (damage) need to be reduced, while the beneficial effects need to be magnified in order to improve the process productivity.

\section{Mathematical model of a single-mass one-sided vibro-impact system}

In the example shown below the dynamics of a single-mass, forcibly damped one-sided vibro-impact oscillator is studied in the case when it is exposed to the action (influence) of the following factors: an elastic force $F_{e}=c_{x} \cdot x$, a viscous damping force $F_{w}=-b_{x} \cdot \dot{x}$, and an external periodic coercive force $F(t)=F_{0} \cdot \cos (\Omega \cdot t+\varphi)$. Thereby, the mass of the oscillator $m$, the spring stiffness (elasticity) $c_{x}$, and the proportionality coefficient $b_{x}$ are considered to have constant values. A single, fixed limiter is located at a distance $x=\Delta$ (Figure 1.); due to this, the system is called one-sided. A positive value of $\Delta$ corresponds to the limiter position presented in Figure 1; on the other hand, the limiter has a negative value when the spring is compressed. The oscillator motion is periodic, i.e. time intervals between two consecutive impacts are the same (identical).

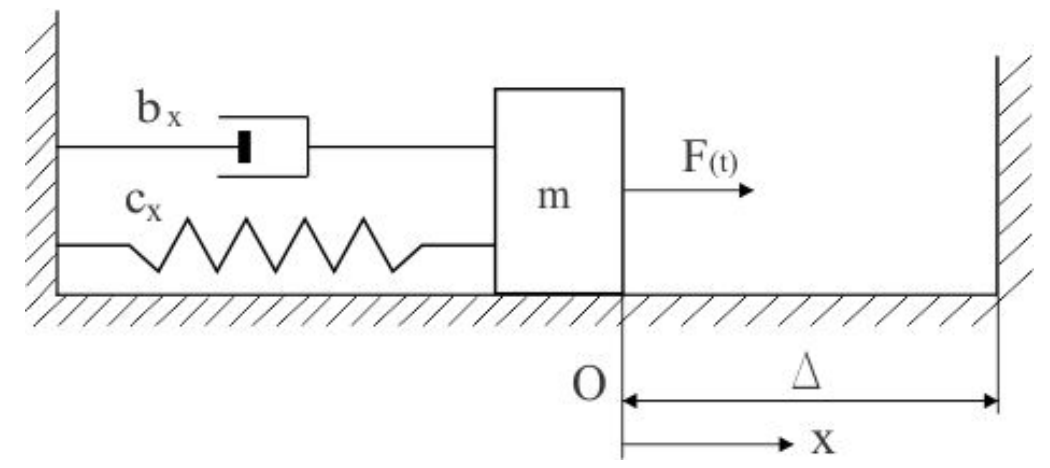

Fig. 1 A mathematical (dynamic) model of the single-mass one-sided impact oscillator.

The differential equation of the system motion at the time intervals between two consecutive impacts has the form of (1) or (2):

$$
m \cdot \ddot{x}+b_{x} \cdot \dot{x}+c_{x} \cdot x=F_{0} \cdot \cos (\Omega \cdot t+\varphi)
$$




$$
\ddot{x}+2 n \cdot \dot{x}+\omega_{x}^{2} \cdot x=P_{1} \cdot \cos (\Omega \cdot t+\varphi)
$$

Where: $n=b_{x} / 2 m$ is the viscous damping coefficient,

$\omega_{x}^{2}=c_{x} / m$ is the angular frequency of free oscillations,

$P_{1}=F_{0} / m$ is the ratio of the external coercive force amplitude and the system mass,

$\Omega$ is the angular frequency of the external coercive force,

$\varphi$ is the initial phase of the external coercive force.

It is known from the differential equation theory that the solution of the homogeneous part of equation (2) has the form of:

$$
x_{h}=e^{-n \cdot t}\left(C_{1} \cdot \cos \left(\sqrt{\omega_{x}^{2}-n^{2}} \cdot t\right)+C_{2} \cdot \sin \left(\sqrt{\omega_{x}^{2}-n^{2}} \cdot t\right)\right)
$$

or $\quad x_{1}=e^{-n \cdot t}\left(C_{1} \cdot \cos \left(\Omega_{1} \cdot t\right)+C_{2} \cdot \sin \left(\Omega_{1} \cdot t\right)\right)$,

where: $\Omega_{1}=\sqrt{\omega_{x}^{2}-n^{2}}$ is the angular frequency of damped oscillations.

The particular solution of the differential equation (2) is taken in the following form: $x_{2}=A \cdot \cos (\Omega \cdot t+\varphi-\varepsilon)$, where A and $\varepsilon$ are unknown costants.

After a few computing operations, one can obtain:

$$
A=\frac{P_{1}}{\sqrt{\left(\omega_{x}^{2}-\Omega^{2}\right)^{2}+4 \cdot n^{2} \cdot \Omega^{2}}} ; \quad \operatorname{tg}(\varepsilon)=\frac{2 n \cdot \Omega}{\omega_{x}^{2}-\Omega^{2}}
$$

The general solution of the linear inhomogeneous differential equation of the second order, with constant coefficients (2), is obtained in the following form: $x=x_{h}+x_{p}=x_{1}+x_{2}$, or:

$$
\begin{aligned}
& x=e^{-n \cdot t}\left(C_{1} \cdot \cos \left(\sqrt{\omega_{x}^{2}-n^{2}} \cdot t\right)+C_{2} \cdot \sin \left(\sqrt{\omega_{x}^{2}-n^{2}} \cdot t\right)\right)+ \\
& +A \cos (\Omega t+\varphi-\varepsilon)
\end{aligned}
$$

Unknown integration constants $C_{1}$ and $C_{2}$ are usually obtained depending on the known initial motion conditions. Since it is not possible to determine in advance the initial conditions under which the oscillator motion will have a periodic character, these constants could not be determined in such a way. Instead of initial motion conditions, conditions of the motion periodicity were used for the determination of constants $C_{1}$ and $C_{2}$; these conditions are defined in accordance with considered model of periodic oscillatory system and are given by expressions (10) and (11) in the continuation of this presentation.

It is necessary to determine what kind of relation between oscillator parameters and parameters of the external coercive force can result in periodic motions in which the period of the oscillator impact at the limiter is equal or proportional to the period of the external coercive force. In the presented analysis it is assumed that the duration of the impact at the limiter is too short in comparision with the period of the oscillator motion between two consecutive impacts; therefore, it is neglected in the calculations.

The effect of the impact is taken into account by the restitution coefficient $0 \leq R \leq 1$. For the plastic impact, $R=0$, while for the absolutely elastic impact it is one, $R=1$. Taking 
into account the aforementioned assumptions, it is sufficient to observe only one interval of the oscillator motion between two consecutive impacts. One impact represents only a part of the consecutive impact process, and the conditions under which any of impacts occurs depend on the conditions under which the previous impact has been performed. Processes consisting of an infinite number of impacts are called infinite impacts. Impacts that are repeated are characteristic of systems with periodic exciting forces; this means that infinite impact processes have a periodic character [1], [2].

In these systems, the oscillator motion period depends on the period of the external coercive force. Usually, there exists an oscillator motion mode with the period which is equal to the external coercive force period, but it is also possible that there exist motion modes with periods which are proportional to the external coercive force period. This possibility of various (multiple) mode existence is the essential characteristic of vibro-impact systems. Due to this, the research into the vibro-impact system dynamics has to involve the definition of all possible types of motion as well as the analysis of the motion stability. This should enable us to extricate and recognize motion modes that can exist (occur) in practice.

In the presented research, a single motion cycle between two consecutive impacts is considered. Thereby, the oscillator velocities before and after the impact are different: $\dot{x}_{-}$is the oscillator velocity before the impact, while $\dot{x}_{+}$is the oscillator velocity after the impact. The relation connecting these two velocities is:

$$
\dot{x}_{+}=-R \cdot \dot{x}_{-}
$$

\section{Conditions for (areas of) the existence of periodic vibro-impact modes (odd, $l=1,3, \ldots$ and even, $l=2,4, \ldots$ ) when $R=0.7$ and $\Delta_{x} \neq 0$}

A periodic motion system is considered and investigated in this paper. It is assumed that the oscillating period is equal or proportional to the external coercive force period. In fact, this means that impacts occur at constant time intervals, $T=2 \cdot \pi / \Omega$, or in proportion to the external coercive force period, $2 \cdot \pi \cdot l / \Omega$, where $l$ is the mode multiplicity.

When $l=1$, then, for a single full period of the external coercive force $F(t)$ variation, a single impact occurs at the fixed limiter. If $l=2$, then, for two full periods $T$ of the external coercive force $F(t)$ variation, a single impact at the limiter occurs again, etc.

One motion cycle is observed, beginning immediately after the impact; the time is measured from that moment until the next impact. When energy losses in time intervals between two consecutive impacts are neglected (which is usually done), the differential equation of the oscillator motion can be expressed in the form of (1) or (2).

If a particular solution is asummed in the form $x_{2}=M \cos (\Omega t+\varphi)+N \sin (\Omega t+\varphi)$, after the first derivative determination and substitution in (2), one can obtain:

$$
x_{2}=\frac{P_{1}\left(\omega_{x}{ }^{2}-\Omega^{2}\right)}{4 n^{2} \cdot \Omega^{2}+\left(\omega_{x}{ }^{2}-\Omega^{2}\right)^{2}} \cdot \cos (\Omega \cdot t+\varphi)+\frac{P_{1} \cdot 2 n \cdot \Omega}{\left(4 n^{2} \cdot \Omega^{2}+\left(\omega_{x}{ }^{2}-\Omega^{2}\right)^{2}\right)} \cdot \sin (\Omega t+\varphi)
$$


The general solution of equation (2), in this case, has the following form:

$$
\begin{aligned}
& x(t)=e^{-n \cdot t}\left(C_{1} \cdot \cos \left(\sqrt{\omega_{x}^{2}-n^{2}} \cdot t\right)+C_{2} \cdot \sin \left(\sqrt{\omega_{x}^{2}-n^{2}} \cdot t\right)\right)+ \\
& +\frac{1-p^{2}}{F(p)} X_{1} \cdot \cos (\Omega \cdot t+\varphi)+\frac{\Delta_{x}}{\pi} \frac{p}{F(p)} X_{1} \cdot \sin (\Omega \cdot t+\varphi)
\end{aligned}
$$

where: $X_{1}=F_{0} / c_{x}$ is the static displacement of the mass under the influence of the force $F_{0}$, $\Delta_{x}=\frac{\pi \cdot b_{x}}{m \cdot \omega_{x}}$ is the damping decrement, $p=\frac{\Omega}{\omega_{x}}$ is the dimensionless frequency, and $\omega_{x}=\sqrt{\frac{c_{x}}{m}}, \quad n=\frac{\omega_{x} \cdot \Delta_{x}}{2 \pi}, \quad F(p)=\left(1-p^{2}\right)^{2}+\left(\frac{\Delta_{x}}{\pi}\right)^{2} p^{2} . \quad$ Introducing $\quad$ replacements $\frac{\left(1-p^{2}\right)}{F(p)} X_{1}=A \cos \varphi_{0}$ and $-\frac{\Delta_{x}}{\pi} \frac{p}{F(p)} X_{1}=A \sin \varphi_{0}$ into expression (8), one obtains:

$$
\begin{aligned}
& x(t)=e^{-n \cdot t}\left(C_{1} \cdot \cos \left(\sqrt{\omega_{x}^{2}-n^{2}} \cdot t\right)+C_{2} \cdot \sin \left(\sqrt{\omega_{x}^{2}-n^{2}} \cdot t\right)\right)+ \\
& +A \cdot \cos \left(\Omega \cdot t+\varphi+\varphi_{0}\right)
\end{aligned}
$$

where: $A=\frac{X_{1}}{F(p)} \sqrt{\left(1-p^{2}\right)^{2}+\left(\frac{\Delta_{x}}{\pi}\right)^{2} p^{2}}, \operatorname{tg}\left(\varphi_{0}\right)=\frac{\frac{\Delta_{x}}{\pi} p}{p^{2}-1}$. follows:

For the considered periodic motion of the system, boundary conditions are assumed as

a) for the beginning of the cycle:

$$
\begin{aligned}
& x(0)=\Delta \\
& \dot{x}(0)=\dot{x}_{+}=-R \dot{x}_{-}
\end{aligned}
$$

b) for the end of the cycle (i.e. for the moment preceding the next impact):

$$
\begin{aligned}
& x\left(\frac{2 \pi \cdot l}{\Omega}\right)=\Delta \\
& \dot{x}\left(\frac{2 \pi \cdot l}{\Omega}\right)=\dot{x}_{-}
\end{aligned}
$$

Since the vibro-impact mode is defined in this manner, it is sufficient to explore a single impact cycle at the interval $\left[0 ; \frac{2 \pi \cdot l}{\Omega}\right]$. Conditions (10) and (11) are adopted based on the fact that velocities preceding the impact have the same values at moments of time $t=0$ and $t=\frac{2 \pi \cdot l}{\Omega}$, i.e. $\dot{x}_{-}(0)=\dot{x}_{-}\left(\frac{2 \pi \cdot l}{\Omega}\right)$. However, in order to realize this mode, it is necessary to use the corresponding external coercive force $F(t)$, i.e. it is necessary to choose the initial phase $\varphi$ of the external coercive force, which allows the realization of the selected mode. 
In practice, periodic modes with a single impact of the mass at the limiter during one period of the external coercive force are mostly used.

Basic characteristics of vibro-impact systems are most clearly expressed with relatively small values of the viscous damping coefficient and the damping decrement.

Based on the expression:

$$
\Omega_{1}=\sqrt{\omega_{x}^{2}-n^{2}}=\sqrt{\omega_{x}^{2}\left(1-\frac{n^{2}}{\omega_{x}^{2}}\right)}=\omega_{x} \sqrt{1-\left(\frac{\Delta_{x}}{2 \pi}\right)^{2}}
$$

it can be adopted that $\Omega_{1} \approx \omega_{x}$.

In addition, if it is assumed that: $\psi=\varphi+\varphi_{0}$, then expression (9) can be written as:

$$
x(t)=e^{-n t}\left(C_{1} \cdot \cos \left(\omega_{x} t\right)+C_{2} \cdot \sin \left(\omega_{x} t\right)\right)+A \cdot \cos (\Omega t+\psi)
$$

The first time derivative of expression (13) represents the velocity:

$$
\begin{aligned}
& \dot{x}=-n e^{-n t}\left(C_{1} \cdot \cos \left(\omega_{x} t\right)+C_{2} \cdot \sin \left(\omega_{x} t\right)\right) \\
& +\omega_{x} e^{-n t}\left(-C_{1} \cdot \sin \left(\omega_{x} t\right)+C_{2} \cdot \cos \left(\omega_{x} t\right)\right)-A \cdot \Omega \cdot \sin (\Omega t+\psi)
\end{aligned}
$$

Now, it is necessary to determine coefficients $C_{1}$ and $C_{2}$. When the boundary conditions (10) and (11) are introduced into expressions (13) and (14), after a few transformations, one can obtain:

$$
\begin{aligned}
& C_{1}=\Delta-A \cdot \cos (\psi) \quad C_{2}=\frac{C_{1} \cdot\left(e^{\frac{\Delta_{x} l}{p}}-\cos \left(\frac{2 \pi \cdot l}{p}\right)\right)}{\sin \left(\frac{2 \pi \cdot l}{p}\right)} \\
& \cos (\psi)=\frac{\Delta}{A}\left(1-\frac{\dot{x}_{-} D}{\omega_{x} \Delta}\right) \\
& \sin (\psi)=\frac{\dot{x}_{-} D}{\omega_{x} A} \cdot B \\
& D=\frac{(R+1) \cdot \sin \left(\frac{2 \pi \cdot l}{p}\right)}{2 \cos \left(\frac{2 \pi \cdot l}{p}\right)-e^{\frac{\Delta_{x} l}{p}}-e^{-\frac{\Delta_{x} l}{p}}} \\
& B=\frac{1}{p} \cdot\left(\frac{R}{D}+\frac{e^{\frac{\Delta_{x} l}{p}}-\cos \left(\frac{2 \pi \cdot l}{p}\right)-\frac{\Delta_{x}}{2 \pi} \sin \left(\frac{2 \pi \cdot l}{p}\right)}{\sin \left(\frac{2 \pi \cdot l}{p}\right)}\right) \\
& \left(\begin{array}{c}
\left.\frac{(2 \pi)}{p}\right) \\
D
\end{array}\right)
\end{aligned}
$$


Whereby, after certain operations and transformations in equation (16), the velocity is obtained as:

$$
\dot{x}_{-}=\frac{1 \pm \sqrt{1-\left(B^{2}+1\right) \cdot\left(1-\frac{A^{2}}{\Delta^{2}}\right)}}{D \cdot\left(B^{2}+1\right)} \cdot \omega_{x} \cdot \Delta
$$

Based on expression (19), it is necessary to explore the areas of vibro-impact mode existence. Primarily, there is a need to determine the area in which the velocity $\dot{x}_{-}$has real values. If it is assumed that the expression under the square root in equation (19) is positive, then $1-\left(1-A^{2} / \Delta^{2}\right) \cdot\left(1+B^{2}\right) \geq 0$. From this, one can obtain the condition which determines the area in which the real values of the velocity $\dot{x}_{-}$are located:

$$
\frac{|\Delta|}{A} \leq \sqrt{1+\frac{1}{B^{2}}}
$$

According to relation (20), vibro-impact modes can exist in the area in which the limiter is placed within the boundaries of the oscillation amplitude of the linear oscillator $0<\Delta<A$, but they can also exist outside that area: $\Delta>A$. In the latter case, when $\Delta>A$, there is a need to provide additional energy for the vibro-impact mode. If condition (20) is not satisfied, vibro-impact modes are not possible.

It is obvious that relation (19) is an ambiguous function of the system parameters; thus, the characteristic of vibro-impact oscillatory systems is the occurence of ambiguous solutions.

In addition, another condition is introduced: the motion trajectory does not exceed the limiter at the interval between two consecutive impacts:

$$
x(t) \leq \Delta
$$

In a general case, it is not possible to determine the areas of vibro-impact mode existence based on condition (21). For systems with a relatively simple structure, only approximate borders of these areas can be determined. To do that, it is primarily important to satisfy the following condition:

$$
\dot{x}_{-} \geq 0
$$

This condition is not applied within boundary conditions and needs to be applied as an additional condition in the motion equation. Let condition (20) be satisfied; it is necessary to determine what kind of restrictions occur due to the need that velocity $\dot{x}_{-}$has a positive value. From relation (19) it follows that if the expression under the square root is greater than 1 , then the velocity $\dot{x}_{-}$has two different values, positive and negative. Due to this, condition (22) is satisfied only for a single value of the velocity $\dot{x}_{-}$. Consequently, by observing equation (19), it is necessary to determine when the velocity is positive, i.e. when condition (22) is satisfied. In that sense, in expression (19), a sign of D should be considered at first. Because D is given by expression (17), both the numerator and the denominator of D have to be observed. The numerator is greater than zero (i.e. positive), when $\sin \left(\frac{2 \pi \cdot l}{p}\right)>0$, and this is for the interval $[2 \pi k ; \pi+2 \pi k]$. This yields: 
$2 \pi k<\frac{2 \pi \cdot l}{p}<\pi+2 \pi k ; 2 \pi k<\frac{2 \pi \cdot l}{p}<\pi(1+2 k) /:(2 \pi \cdot l) ; \frac{k}{l}<\frac{1}{p}<\frac{1+2 k}{2 l} ;$ the reciprocal is

$$
\frac{2 l}{1+2 k}<p<\frac{l}{k}
$$

The numerator is less than zero (i.e. negative), when $\sin \left(\frac{2 \pi \cdot l}{p}\right)<0$, and this is for the interval $[\pi+2 \pi k ; 2 \pi+2 \pi k]$. This yields:

$\pi+2 \pi k<\frac{2 \pi \cdot l}{p}<2 \pi+2 \pi k ; \pi(1+2 k)<\frac{2 \pi \cdot l}{p}<2 \pi(1+k) /:(2 \pi \cdot l), \frac{1+2 k}{2 l}<\frac{1}{p}<\frac{1+k}{l} ;$

the reciprocal is

$$
\frac{l}{1+k}<p<\frac{2 l}{1+2 k}
$$

Particular attention should be paid to the denominator in expression (17). If both the numerator and the denominator have the same signs, then the ratio $\Delta / D$ is always positive. Due to a small value of the damping decrement $\Delta_{x}$, values $e^{\frac{\Delta_{x} l}{p}}$ and $e^{-\frac{\Delta_{x} l}{p}}$ in (17) are approximately equal to 1 . The expression in the denominator in (17) is $2 \cos \left(\frac{2 \pi \cdot l}{p}\right)-2<0$ : its value is less than zero throughout the whole interval of variable $p$. This assumption is proven by the diagram of the denominator function in expression (17): $f(p)=2 \cos \left(\frac{2 \pi \cdot l}{p}\right)-e^{\frac{\Delta_{x} l}{p}}-e^{-\frac{\Delta_{x} l}{p}}$ (Figure 2, a and b).

This diagram is obtained using the programs Wolfram Mathematica 7 (Figure 2a) and Mathcad 14 (Figure 2b), when $l=1$ and $\Delta_{x}=0.2 \pi$. It can be seen from this diagram that when $p \rightarrow \infty$, the value of $f(p)$ tends to the x-axis, but it does not cut it, while the denominator is negative in the whole interval $p \in[0 ; \infty)$.

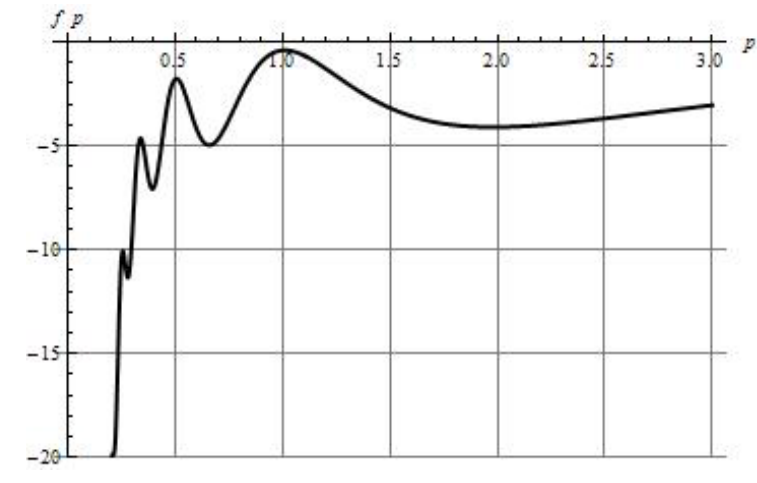

(a)

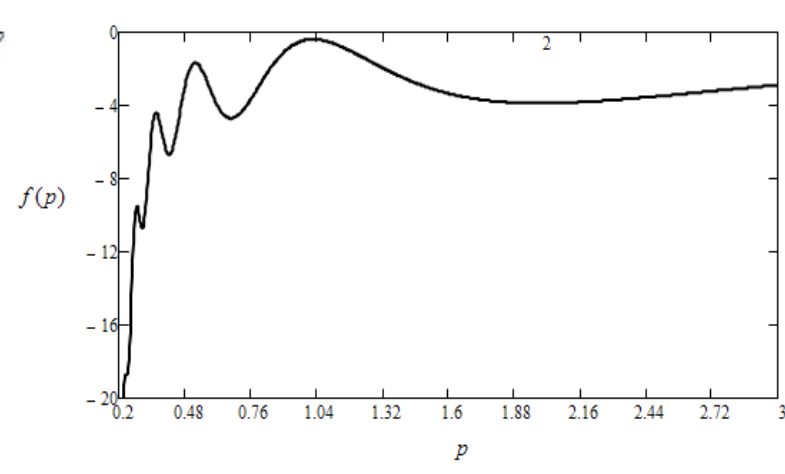

(b)

Fig. 2 a, b The diagram of the denominator function $f(p)$ from expression (17). 


\subsection{Mode I}

The analysis starts with the mode I which corresponds to the positive sign in front of the square root in expression (19). The initial velocity $\dot{x}_{-}$, in this case, is always positive in area (20), even when is $\Delta / D>0$ in expression (19). In this case, the condition that the expression under the square root has to be greater than or equal to zero needs to be satisfied so that the velocity could have real values:

$$
1-\left(1-A^{2} / \Delta^{2}\right) \cdot\left(1+B^{2}\right) \geq 0
$$

After a few simple calculations, the following is obtained:

$$
\frac{A^{2}}{\Delta^{2}} \geq \frac{B^{2}}{1+B^{2}} \text {. }
$$

If the reciprocal is taken, the sign changes again:

$\frac{\Delta^{2}}{A^{2}} \leq \frac{B^{2}+1}{B^{2}}$, i.e. $\frac{\Delta^{2}}{A^{2}} \leq 1+\frac{1}{B^{2}}$, and after the root operation, the following is obtained:

$$
\frac{|\Delta|}{A} \leq \sqrt{1+\frac{1}{B^{2}}} \text {. }
$$

The latter relation represents previously presented expression (20), which is the first condition determining the area in which real values of the velocity $\dot{x}_{-}$are located.

Now, it is necessary to explore the second condition, which is presented by equation (22) and which defines the condition that the velocity has to be positive, $\dot{x}_{-} \geq 0$.

Due to the fact that, for this mode, the sign in front of the square root in (19) has to be positive, it is necessary to observe the sign of $D$ in equation (17).

Because the expression for $D$ needs to be positive (consequently, the velocity is also positive), it is necessary that both the numerator and the denominator have the same signs (positive or negative). The denominator in $D$ is always negative, $f(p)<0$, which is shown by Figures 2 a, b. Due to this, the numerator in $D$ has to be negative too because, in that way, the expression for $D$ has a positive value.

Previously it has been shown that the numerator is negative when $\sin \left(\frac{2 \pi \cdot l}{p}\right)<0$; in that case the interval $\frac{l}{1+k}<p<\frac{2 l}{1+2 k}$, (24), was obtained. Then, the following is valid: $(\Delta / D>0$, i.e. $D>0)$.

This means that interval (24) is the condition for the mode I existence, while it has already been shown that expression (20) is the condition for the existence of the same mode.

Therefore, in this way, the vibro-impact mode I is described, and it is defined by the positive sign in front of the square root in expression (19).

In addition, from interval (24), one obtains that $1 \leq p<2$ for $l=1,2 \leq p<4$ for $l=2$, and $3 \leq p<6$ for $l=3$, etc. This is shown by Figures $3 \mathrm{a}, \mathrm{b}$, and $\mathrm{c}$ which represent the areas where vibro-impacts exist for the above listed values of parameter $l$.

\subsection{Mode II}

The mode II which corresponds to the negative sign in front of the square root in expression (19) is also explored. 
First, the case when $\Delta / D>0$, which is the condition for the velocity being positive, is explored. In this mode, one takes the negative sign in front of the square root in (19) (for the mode I, this sign was positive). Thus, the expression under the square root has to be less than 1 because, in that case, the numerator in expression (19) is positive:

$$
1-\left(1-A^{2} / \Delta^{2}\right) \cdot\left(1+B^{2}\right)<1
$$

After a few calculations, it becomes $A^{2} / \Delta^{2}<1$

If the reciprocal is taken, the sign changes again: $\Delta^{2} / A^{2}>1,|\Delta|>A$. This leads to the solution $|\Delta|>A$. This solution does not satisfy the condition $x(t) \leq \Delta,(21)$, and thus, it is not suitable for further investigation because, in that case, a mode without an impact can exist, but also a mode with an impact (Figures $3 \mathrm{a}, \mathrm{b}, \mathrm{c}$ ). The mode with an impact can exist only when additional energy is delivered to the system, i.e. when the amplitude of the external coercive force $F(t)$ is increased.

Thus, the vibro-impact mode II is described; it is defined by the negative sign in front of the square root in expression (19).

\subsection{Mode III}

At last, the mode III, which corresponds to a negative sign in front of the square root in expression (19), is explored. However, unlike the mode II, here the case $\Delta / D<0$ has to be explored (for mode II, it is the case $\Delta / D>0$ ) so that the velocity is positive. In accordance with this fact, the expression under the square root in (19) has to be greater than 1 , which is in contrast with the mode II. Due to this, the numerator in expression (19) has to be negative. Therefore, if the velocity is to be positive, it is required that $\Delta / D<0$, i.e. $D<0$. In other words, in this case, both the numerator and the denominator in expression (19) are negative, and thus, the velocity is positive. It has been shown previously that $\mathrm{D}$ is negative when the numerator in (17) is positive, because its denominator $f(p)$ is always negative, and this holds for interval (23), $\frac{2 l}{1+2 k}<p<\frac{l}{k}$.

Hence, in order to have a positive velocity in mode III, it is necessary to explore the case when the expression under the square root in (19) is greater than 1. In this case, from (19) one can obtain the following expression:

$$
1-\left(1-A^{2} / \Delta^{2}\right) \cdot\left(1+B^{2}\right)>1
$$

After a few calculations, it becomes: $A^{2} / \Delta^{2}>1$

If the reciprocal is taken, the sign changes again: $\Delta^{2}<A^{2},|\Delta|<A$.

Thus, both conditions are satisfied: $|\Delta|<A$ and the velocity is positive. In addition, from interval (23), one obtains $p \geq 2$ for $l=1$, then $p \geq 4$ for $l=2$ and $p \geq 6$ for $l=3$. This is shown by Figures 3 a, b, c which represents the areas in which vibro-impacts exist for the above listed values of parameter $l$.

Based on the analysis of the presented three modes, it can be concluded that in the frequency interval $p$, for all real values of parameters which satisfy the condition $\Delta<A$, vibro-impact modes can exist. For $\Delta=A$, the velocity $\dot{x}_{-}$is equal to zero, which corresponds to the oscillator motion, ending only with the touch of the limiter (without impact). The conducted research has shown that vibro-impact modes can exist even in the case when $\Delta>A$. 


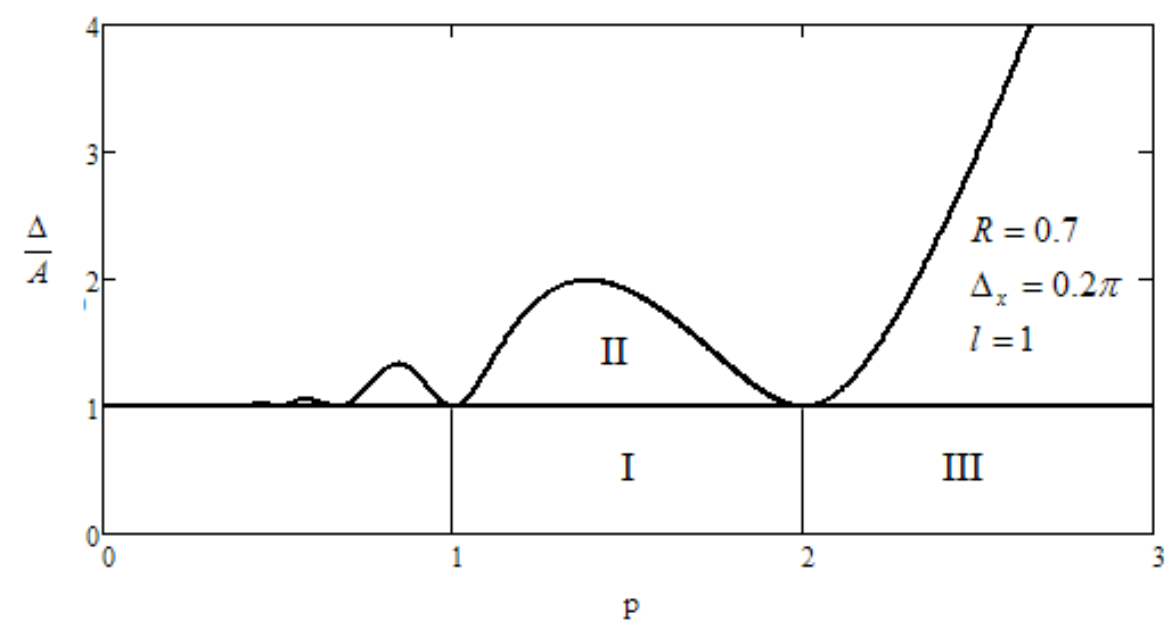

(a)

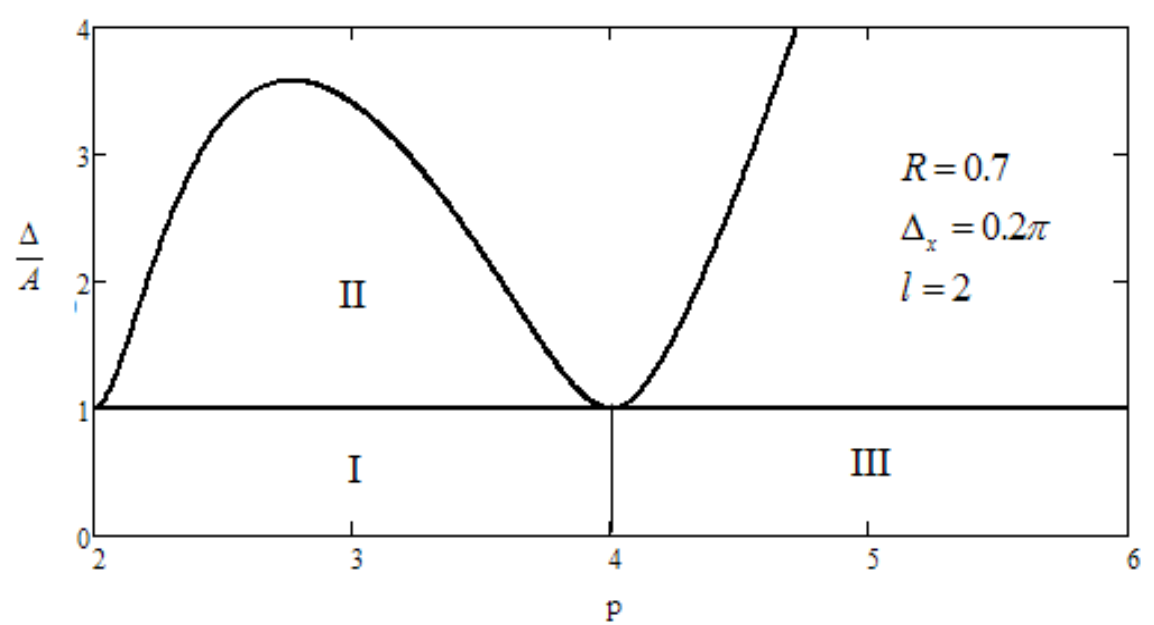

(b)

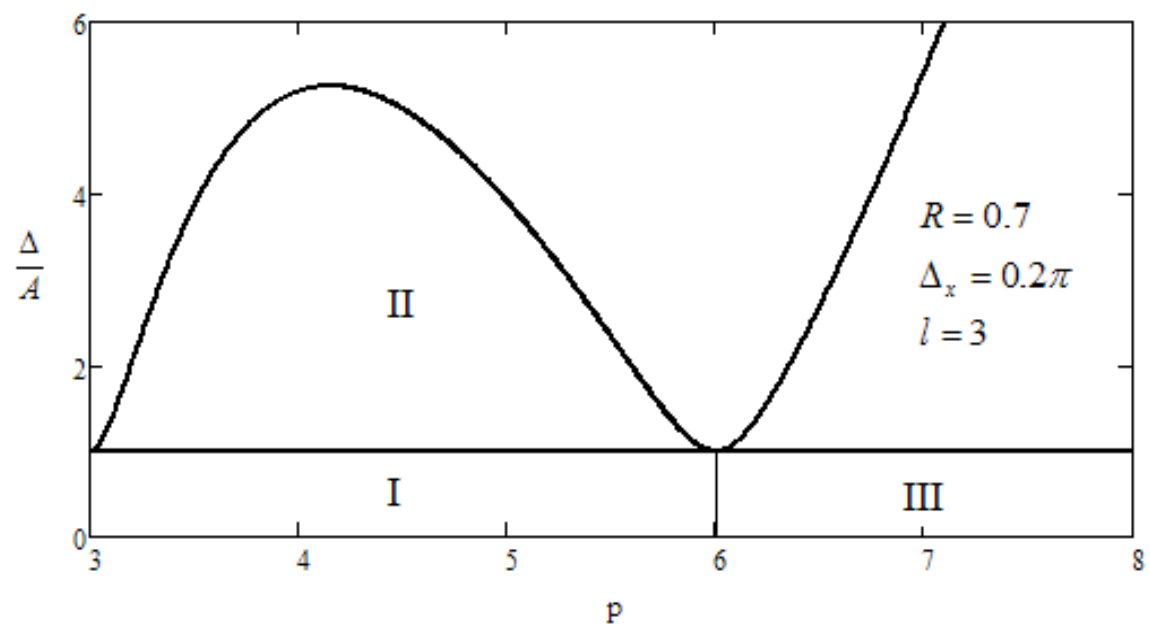

(c)

Fig. 3 a, b, c Areas of vibro-impact mode I, II, and III existence.

Figures 3 a, b, c show the areas of vibro-impact mode I, II, and III exist. Diagrams are plotted by using software programmes Mathcad 14 and Wolfram Mathematica 7, using expression (20), for the constant values of $R$ and $\Delta_{x}$, and for the corresponding values of $l$ 
$(l=1,2$, and 3$)$. These figures clearly show that, by increasing multiplicity $l$, areas of mode existence increase, too, i.e. become wider. Diagrams in Figures 3a, b, c enable us to determine and define the frequency interval of the vibro-impact process, when the value of the distance $\Delta$ is known. These diagrams also show vibro-impact mode existence, when the distance $\Delta$ is greater than the oscillation amplitude, $\Delta>A$, (mode II).

In addition, expressions for the denominator $f(p)$ of equation (17) are presented with corresponding diagrams drawn by the mathematic package Wolfram Mathematica 7 (Figures $4 \mathrm{a}, \mathrm{b}, \mathrm{c}$.). At the end of the computation, completely the same diagrams as in Figures $3 \mathrm{a}, \mathrm{b}, \mathrm{c}$ are obtained for the areas where vibro-impact modes exist when $l=1, l=2$, and $l=3$.

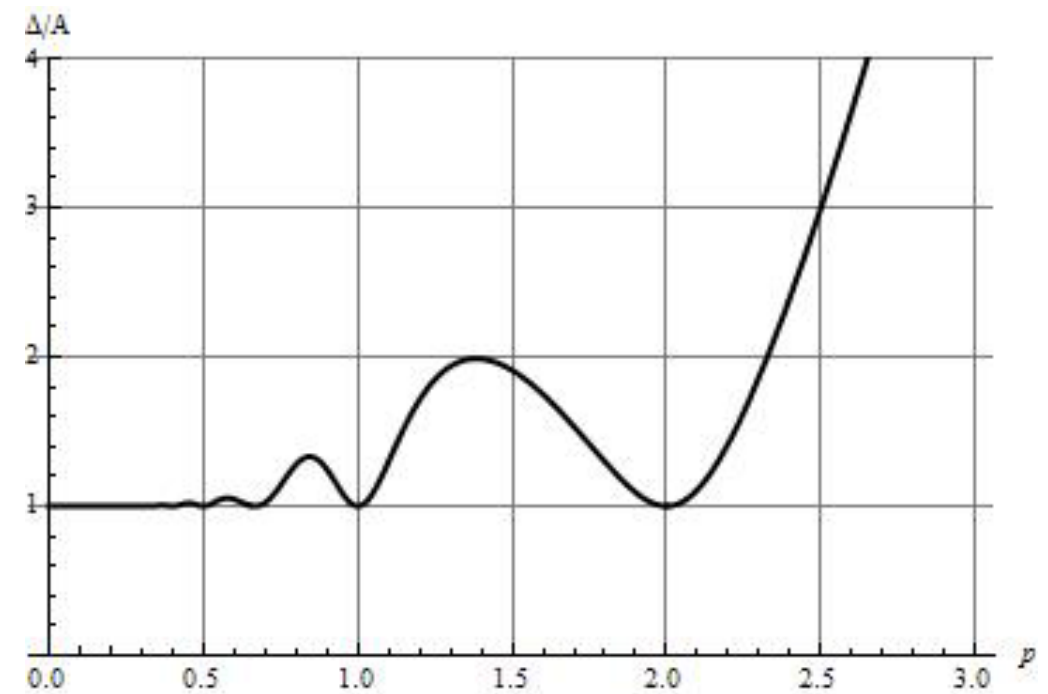

(a)

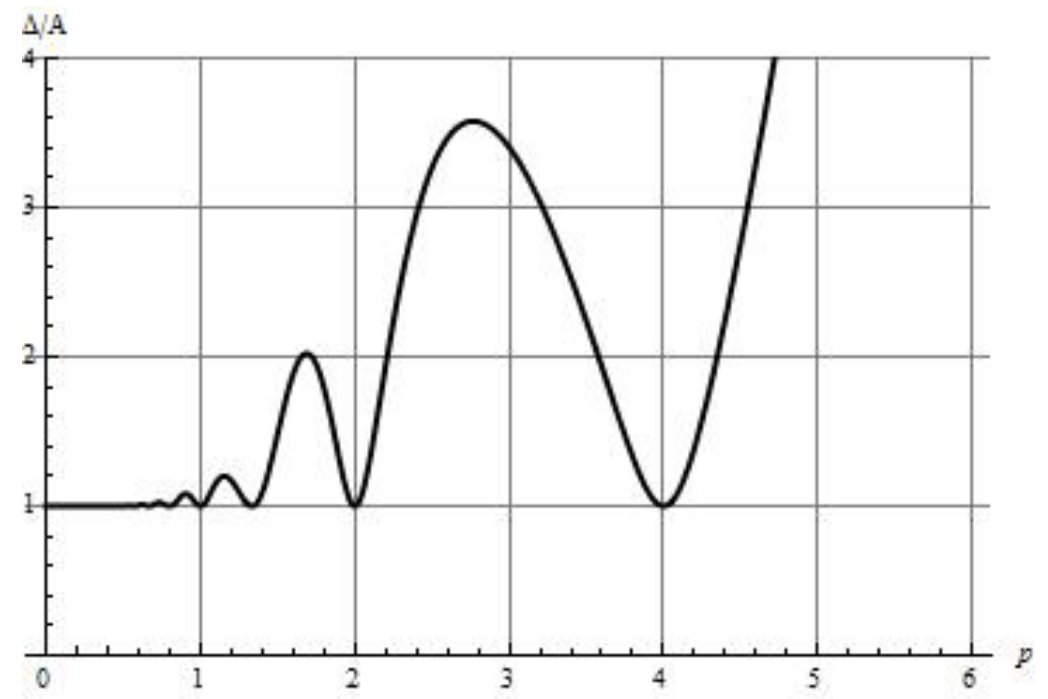

(b) 


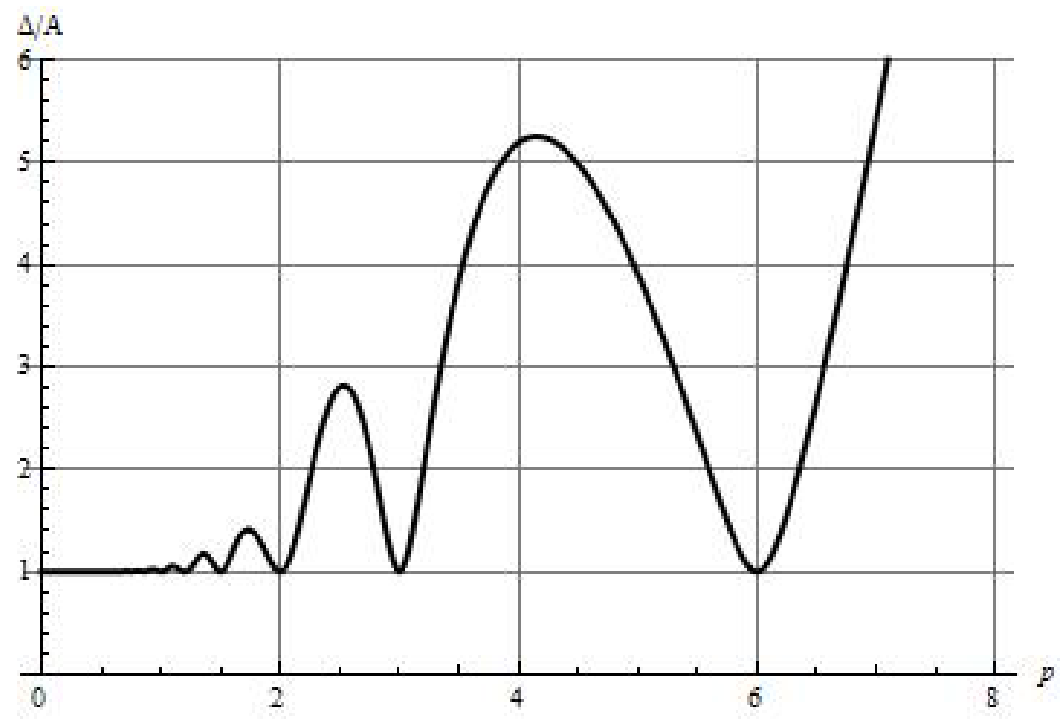

(c)

Fig. 4 a, b, c Areas of vibro-impact mode I, II, and III existence.

Figure 5 shows a phase diagram drawn by Mathcad 14 for two different values of the damping decrement, $\Delta_{x}=0.1 \pi$ and $\Delta_{x}=0.2 \pi$, when the following parameters are known: $F_{0}=2 N, X_{1}=2 N, m=1 \mathrm{~kg}, \omega_{x}=1 \mathrm{~s}^{-1}, \Delta=1 \mathrm{~m}, R=0.7, p=1.5, \Omega=1.5 \mathrm{~s}^{-1}$.

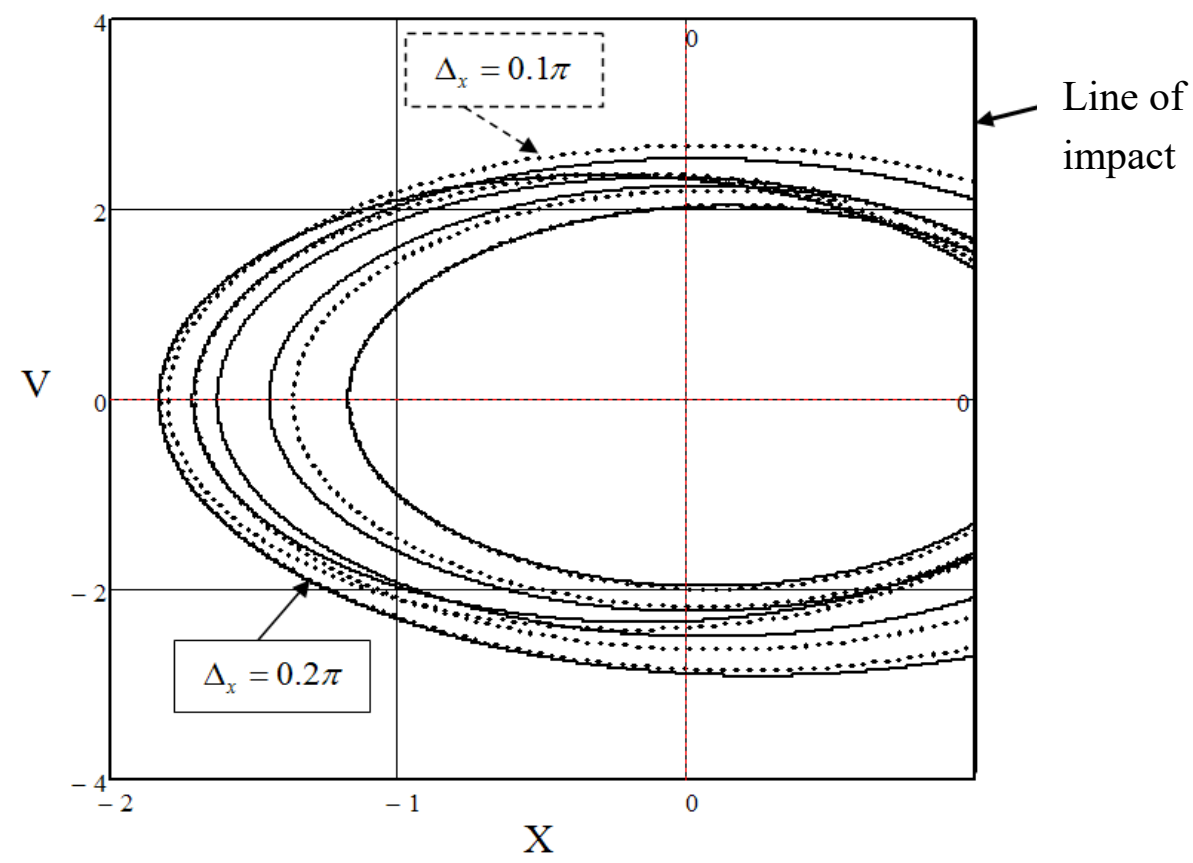

Fig. 5 Phase portrait of the time interval $\mathrm{T}$ between two consecutive impacts.

\section{Conditions for (areas of) the periodical vibro-impact mode existence}

(odd, $l=1,3, \ldots$ and even, $l=2,4, \ldots$ ) when $R=1$ and $\Delta_{x}=0$.

New (additional) results, giving more information about the areas in which vibro-impact modes exist, are very significant. In that sense, a case of the absolutely elastic impact $(R=1)$, with a damping decrement of $\Delta_{x}=0$, is explored. In this case, the following is obtained from relations (17) and (18): 


$$
\begin{aligned}
& D= \frac{2 \cdot \sin \left(\frac{2 \pi \cdot l}{p}\right)}{2 \cos \left(\frac{2 \pi \cdot l}{p}\right)-2} \\
& B=\left(\frac{1}{D}+\frac{1-\cos \left(\frac{2 \pi \cdot l}{p}\right)}{\sin \left(\frac{2 \pi \cdot l}{p}\right)}\right) \frac{1}{p}=\left(\frac{\cos \left(\frac{2 \pi \cdot l}{p}\right)-1}{\sin \left(\frac{2 \pi \cdot l}{p}\right)}+\frac{1-\cos \left(\frac{2 \pi \cdot l}{p}\right)}{\sin \left(\frac{2 \pi \cdot l}{p}\right)}\right) \frac{1}{p}=0
\end{aligned}
$$

Carrying out certain operations and transformations in (19), the velocity becomes:

$$
\dot{x}_{-}=\frac{\left(1 \pm \sqrt{1-\left(1-A^{2} / \Delta^{2}\right) \cdot\left(1+B^{2}\right)}\right) \cdot \omega_{x} \cdot \Delta}{D \cdot\left(1+B^{2}\right)}=\ldots=\frac{\omega_{x} \cdot \Delta}{D}\left(1 \pm \frac{A}{|\Delta|}\right)
$$

After corresponding substitutions in (13), the following law of the system motion is obtained:

$$
x(t)=\frac{\Delta-A \cdot \cos (\psi)}{\cos \left(\frac{\pi \cdot l}{p}\right)} \cdot\left(\cos \left(\omega_{x} \cdot t-\frac{\pi \cdot l}{p}\right)\right)+A \cdot \cos (\Omega \cdot t+\psi)
$$

The extreme value is obtained for: $\cos (\psi)= \pm 1$, i.e. when: $\omega_{x} \cdot t-\frac{\pi \cdot l}{p}=0$, from where the following is obtained: $t=\frac{\pi \cdot l}{p \cdot \omega_{x}}=\frac{\pi \cdot l}{\frac{\Omega}{\omega_{x}} \cdot \omega_{x}}=\frac{\pi \cdot l}{\Omega}$, i.e. $t=\frac{\pi \cdot l}{\Omega}$.

It is necessary to satisfy condition (21), and, in that way, expression (30) becomes:

$$
x\left(\frac{\pi \cdot l}{\Omega}\right)=\frac{\Delta-A \cdot \cos \psi}{\cos \left(\frac{\pi \cdot l}{p}\right)}+A \cdot(-1)^{l} \cdot \cos (\psi) \leq \Delta
$$

The expression for the velocity (29) is substituted in equation (16), resulting in:

$$
\cos (\psi)=\frac{\Delta}{A}\left(1-\frac{\dot{x}_{-} D}{\omega_{x} \Delta}\right)=\frac{\Delta}{A}\left(1-\frac{\omega_{x} \Delta}{D}\left(1 \pm \frac{A}{|\Delta|}\right) \frac{D}{\omega_{x} \Delta}\right)=\frac{\Delta}{A}\left(1-\left(1 \pm \frac{A}{|\Delta|}\right)\right)=\frac{\Delta}{A}\left(\mp \frac{A}{|\Delta|}\right)=\frac{1}{A}\left(\mp A \frac{\Delta}{|\Delta|}\right)
$$

Finally, one obtains the following:

$$
A \cdot \cos (\psi)=\mp A \frac{\Delta}{|\Delta|}
$$

Taking into account expression (32), after corresponding transformations in inequality (31), the following conditions are obtained:

a) When $l$ is even $(l=2,4,6, \ldots): \frac{\Delta-A \cdot \cos \psi}{\cos \left(\frac{\pi \cdot l}{p}\right)}+A \cdot(-1)^{l} \cdot \cos (\psi) \leq \Delta$; 
Finally, one obtains:

$$
\begin{aligned}
& \frac{|\Delta|}{A}<\mp 1 \text {, when it is : } \cos \left(\frac{\pi \cdot l}{p}\right)<1 \\
& \text { and } \frac{|\Delta|}{A}>\mp 1 \text {, when it is }: \cos \left(\frac{\pi \cdot l}{p}\right)>1
\end{aligned}
$$

b) When $l$ is odd $(l=1,3,5, \ldots): \frac{\Delta-A \cdot \cos \psi}{\cos \left(\frac{\pi \cdot l}{p}\right)}+A \cdot(-1)^{l} \cdot \cos (\psi) \leq \Delta$;

Finally, the following is obtained:

$$
\begin{aligned}
& \frac{|\Delta|}{A}<\mp \operatorname{ctg}^{2}\left(\frac{\pi \cdot l}{2 p}\right) \text {, when it is : cos }\left(\frac{\pi \cdot l}{p}\right)>0 \\
& \text { and } \frac{|\Delta|}{A}>\mp \operatorname{ctg}^{2}\left(\frac{\pi \cdot l}{2 p}\right) \text {, when it is }: \cos \left(\frac{\pi \cdot l}{p}\right)<0
\end{aligned}
$$

In the observed and explored vibro-impact process, the oscillation centre moves due to asymmetric one-sided impacts. The oscillation centre is marked by the point $\mathrm{O}$ (Figure 1), and the oscillator motion should be the same (symmetric) on both sides (left and right) of the point O. However, oscillations are not symmetric because the mass impacts the limiter on the right side, while, on the left side, the mass moves without any impact.

\section{Conclusion}

Analysis of the horizontal straight-line motion of a single-mass, one-sided (with a single limiter) vibro-impact oscillator is presented in this paper. The oscillator motion is periodic, with the constant period $T$, which represents the time interval between two consecutive impacts. The forcibly damped impact oscillator is exposed to the action of the following factors: an elastic force $F_{e}$, a viscous damping force $F_{w}$, and an external periodic coercive force $F(t)$. Two cases are explored: the first case, when the restitution coefficient is $R=0.7$ and the damping decrement is $\Delta_{x} \neq 0$, and the second case, when $R=1$ and $\Delta_{x}=0$.

In the first case, the areas of periodical vibro-impact mode existence are explored, when the mode multiplicity $l$ has both even and odd values. The obtained results are presented in Figure 3 ( $a, b$, and $c)$, showing the existence of three vibro-impact areas. It can be clearly seen that, in a general case, when the mode multiplicity $l$ has a greater value, the areas of vibroimpact mode existence are wider.

In the second case, the areas of periodical vibro-impact mode existence, when the mode multiplicity $l$ has both even and odd values, are explored and the obtained results are presented by corresponding expressions.

For the calculation and graphic visualization of the vibro-impact system dynamics, mathematical software packages Mathcad 14 and Wolfram Mathematica 7 were used.

This paper presents the results obtained for a defined form of the vibro-impact system structure (with a single-mass, a single limiter and one spring), which is exposed to the influence of a periodic external force. Nevertheless, the obtained results can give some ideas and guideposts for research into vibro-impact systems with different structures and different types of external force. 
The main result and the conclusion of the performed research is the following: when the viscous damping coefficient has a smaller value, the areas of vibro-impact mode existence become wider. This means that damping decreases the areas in which vibro-impact modes exist, but it can not cause the total disappearance of vibro-impacts. When technical (working) conditions allow, the efficient solution to potential problems is to increase the distance $\Delta$ from the limiter beyond the boundaries of existence of a given vibro-impact mode. Such a structure is realized on the basis of amplitude-frequency characteristics of the vibro-impact system presented by Figure 3 ( $a, b$, and $c)$.

\section{REFERENCES}

[1] Babicki V. I., Theory of Vibro-Impact Systems and Applications, Springer-Verlag, Berlin, 1998. (Revised translation on English from Russian, Nauka, Moscow, 1978.).

[2] Jakovlevic M. I., Elementi teorii optimalnogo upravlenija periodičeskimi režimami vibroudarnih sistem: Sistemi, linejnie v promežutkah meždu soudareniami, Moskva, 2010.

[3] Beards C. E., Structural Vibration: Analysis and Damping, University of London, 1996.

[4] Gendelman O.V., Alloni A., Dynamics of forced system with vibro-impact energy sink, Journal of Sound and Vibration, Volume 358, 2015, Pages 301-314. https://doi.org/10.1016/j.jsv.2015.08.020

[5] M. Xu , Y. Wang, X. L. Jin, Z. L. Huang, T. X. Yu, Random response of vibro-impact systems with inelastic contact, International Journal of Non-Linear Mechanics, 2013. https://doi.org/10.1016/j.ijnonlinmec.2012.12.010

[6] Jingyue Wang, Haotian Wang, and Tie Wang, External Periodic Force Control of a Single-Degree-ofFreedom Vibroimpact System, Journal of Control Science and Engineering, Volume 2013.

[7] Perchikov N., Gendelman O.V., Dynamics and stability of a discrete breather in a harmonically excited chain with vibro-impact on-site potential, Physica D: Nonlinear Phenomena, 2015, Volumes 292, p 8-28. https://doi.org/10.1016/j.physd.2014.10.009

[8] Kember S.A., Babickij V.I., Excitation of vibro impact system by periodic impulses, Journal of Sound and Vibration; 1999, Vol. 227. https://doi.org/10.1006/jsvi.1999.2353

[9] Yang Liu, Ekaterina Pavlovskaia, Marian Wiercigroch, ZhikePeng, Forward and backward motion control of a vibro-impact capsule system, International Journal of Non-Linear Mechanics, 2015, p 30-46. https://doi.org/10.1016/j.ijnonlinmec.2014.10.009

[10] Liviu Brindeu, Stability of the periodic motions of the vibro-impact systems, Chaos, Solitons and Fractals 11, 2000, Pages 2493-2503. https://doi.org/10.1016/S0960-0779(99)00195-2

[11] Silvio L.T. de Souza, Ibere L. Caldas, Controlling chaotic orbits in mechanical systems with impacts, Chaos, Solitons and Fractals 19, 2004, Pages 171-178. https://doi.org/10.1016/S0960-0779(03)00129-2

[12] Jian Mao, Yu Fu, Peichao Li, Dynamics of periodic impulsive collision in escapement mechanism, Shock and Vibration 20, 2013, Pages 1001-1010. https://doi.org/10.1155/2013/350429

Submitted: $\quad 10.2 .2017$

Accepted: $\quad 20.4 .2017$

\author{
Ljubiša Garić \\ Teaching assistant \\ Department of Mechanical Engineering \\ Faculty of Technical Sciences \\ University of Priština \\ 38220 Kosovska Mitrovica \\ Kneza Miloša 7 \\ Fax/tel: +38128425321 \\ ljubisa.garic@pr.ac.rs
}

